\title{
BMJ Global Health Using a cross-contextual reciprocal learning approach in a multisite implementation research project to improve self-management for type 2 diabetes
}

Josefien van Olmen, ${ }^{1,2}$ Peter Delobelle, ${ }^{3,4}$ David Guwatudde ${ }^{5}$ Pilvikki Absetz, ${ }^{6,7}$ David Sanders, ${ }^{8}$ Helle Mölsted Alvesson, ${ }^{9}$ Thandi Puoane, ${ }^{4}$ Claes-Goran Ostenson, ${ }^{10}$ Göran Tomson, ${ }^{11,12}$ Roy William Mayega, ${ }^{5}$ Carl Johan Sundberg, ${ }^{12,13}$ Stefan Peterson, ${ }^{9,14}$ Meena Daivadanam ${ }^{9,15}$

To cite: van Olmen J, Delobelle P, Guwatudde D, et al. Using a cross-contextual reciprocal learning approach in a multisite implementation research project to improve self-management for type 2 diabetes. BMJ Glob Health 2018;3:e001068. doi:10.1136/ bmjgh-2018-001068

Handling editor Seye Abimbola

Jv0 and PD are joint first authors.

SP and MD are joint senior authors.

Received 19 July 2018 Revised 23 October 2018 Accepted 28 0ctober 2018

\section{Check for updates}

\section{Author(s) (or their} employer(s)) 2018. Re-use permitted under CC BY-NC. No commercial re-use. See rights and permissions. Published by BMJ.

For numbered affiliations see end of article.

Correspondence to Dr Josefien van Olmen; jvanolmen@itg.be

\section{ABSTRACT}

This paper reports on the use of reciprocal learning for identifying, adopting and adapting a type 2 diabetes self-management support intervention in a multisite implementation trial conducted in a rural setting in a low-income country (Uganda), a periurban township in a middle-income country (South Africa) and socioeconomically disadvantaged suburbs in a highincome country (Sweden). The learning process was guided by a framework for knowledge translation and structured into three learning cycles, allowing for a balance between evidence, stakeholder interaction and contextual adaptation. Key factors included commitment, common goals, leadership and partnerships. Synergistic outcomes were the cocreation of knowledge, interventions and implementation methods, including reverse innovations such as adaption of community-linked models of care. Contextualisation was achieved by cross-site exchanges and local stakeholder interaction to balance intervention fidelity with local adaptation. Interdisciplinary and crosssite collaboration resulted in the establishment of learning networks. Limitations of reciprocal learning relate to the complexity of the process with unpredictable outcomes and the limited generalisability of results.

\section{INTRODUCTION}

The rising burden of non-communicable diseases (NCDs) worldwide and its clustering in vulnerable and disadvantaged groups, as well as the insufficient response of healthcare systems, highlight the need for shared learning across contexts on innovative approaches to prevention and care. This is especially relevant given the 2030 Agenda with its 17 Sustainable Development Goals, which offer a framework for development that has been accepted globally. ${ }^{1}$

\section{Summary box}

- This paper offers a methodology for researchers to facilitate reciprocal learning across contexts and disciplines from planning to implementation of innovations within complex multisite implementation research projects.

- Using a framework has an added value to guide the collaboration and keep the balance between implementation fidelity and flexibility.

- Reciprocal learning facilitates contextualisation, but the process needs careful management with due attention to output and relations.

- In the SMART2D (Self-Management Approach and Reciprocal learning for Type 2 Diabetes) project, this resulted in the identification, adoption and adaptation of a type 2 diabetes self-management support intervention in three vastly different income settings and groups.

Type 2 diabetes (T2D), in particular, poses a challenge for societies worldwide, with age-standardised prevalence rates in 2014 of $9.0 \%$ for men and $7.9 \%$ for women. ${ }^{2}$ The burden of disease and its consequences are concentrated in low-income and middle-income countries (LMICs), with a prevalence of $12.3 \%$ in low-income countries, ${ }^{3}$ where health systems are not adapted to the growing number of people with T2D and other chronic conditions. In high-income countries (HICs), which focus on professional, facility-based care, T2D prevalence among disadvantaged population groups is also higher than in the general population, and outcomes are significantly affected by differences in race, ethnicity and social determinants of health. ${ }^{4}$ Health centres in vulnerable areas experience a 
higher patient load, with prevention efforts not reaching those in greatest need of intervention. ${ }^{5}$

Chronic care models and guidelines for diabetes care and self-management support exist, but they often focus on HICs and default professional-based care.$^{6-8}$ The approaches typically emphasise individual responsibility of the person with diabetes and ignore the more upstream determinants of health behaviour, ${ }^{9}$ and despite the awareness of the role of the family and community in self-management support there is limited scale-up of community-based approaches globally. ${ }^{10}$

The global challenge of diabetes and the commonalities in health system constraints in HIC and LMIC call for cross-contextual research ${ }^{11}$ and for new collaboration models based on reciprocal learning, such as those developed for other global health challenges like antimicrobial resistance. ${ }^{12}$ Reciprocal learning as a learning partnership is characterised by a shared, back-and-forth process between team members in which each partner is both the learner and the coach, ${ }^{1314}$ which has shown in small organisations to be correlated with better implementation. ${ }^{15}$ Although such innovative partnerships are promoted by new funding opportunities, their implementation and effectiveness are not self-evident. ${ }^{16}$

This paper answers the call for cross-contextual learning to advance implementation research on T2D and other chronic diseases, by describing a research project that prioritised reciprocal learning as an approach and outcome. The last decades have witnessed a rising interest in this type of research, but little is known about the barriers and facilitators of implementation. The aim of this paper is to analyse the process and methodology of reciprocal learning, by providing a summary of the method, learning cycles and lessons learnt so far in this project.

\section{PROGRAMME DESCRIPTION The SMART2D project}

The 'Self-Management Approach and Reciprocal learning for Type 2 Diabetes' (SMART2D) research project was established in 2014 following a European Union H2020 call for implementation research. The collaboration included anthropologists, behavioural scientists, epidemiologists, diabetologists and health systems experts, who worked in three different settings: a rural area in Uganda (low-income country), a semiurban township in Cape Town, South Africa (middle-income country) and socioeconomically disadvantaged suburbs with a high proportion of immigrants in Stockholm, Sweden (HIC). These settings were purposefully selected because of their differences but also opportunities in terms of reciprocal learning (box 1).

Each context has particular strengths: experience with community involvement in HIV/AIDS/tuberculosis care in Uganda and South Africa ${ }^{17}{ }^{18}$; evidence-based models for integrated NCD care in South Africa ${ }^{19}$; and strong facility-based diabetes care and NCD prevention tools in

\section{Box 1 Study context of SMART2D}

- Uganda: Measured country-wide diabetes prevalence is approximately $1.4 \%,{ }^{44}$ but national age-adjusted prevalence rates are higher, reaching $4.4 \%(2.1 \%-7.8 \%)$ for men and $4.7 \%(2.3 \%-8.2 \%)$ for women in $2014 .{ }^{45}$ Diabetes services are offered in larger health centres and district hospitals and are constrained by inadequate resources, resulting in shortages of medicines and diagnostics, insufficiently detailed care guidelines and lack of standardised strategies for patient follow-up. Like other low-income countries, Uganda has extended its delivery of health services into the community by collaborating with village health teams and involving patient peer groups for HIV/AIDS care. ${ }^{46}$

- South Africa: The age-adjusted prevalence rate of diabetes in 2014 was $9.7 \%(4.9 \%-16.3 \%)$ for men and $12.6 \%(6.8 \%-20.2 \%)$ for women, ${ }^{45}$ with variations across ethnic groups and a rapid increase among urban-dwelling black South Africans. ${ }^{47}$ While there are comprehensive diabetic services in South Africa, coverage is not universal and services are of varying quality and generally perceived to be poor, especially in the public healthcare system. ${ }^{48}$ National guidelines for type 2 diabetes management for patient education, lifestyle changes and pharmacotherapy exist, ${ }^{49}$ but are not well followed in practice. ${ }^{48} 50$

- Sweden: The age-adjusted prevalence of diabetes in 2016 was $5.8 \%(3.2 \%-9.3 \%)$ for men and $4.0 \%(2.1 \%-6.6 \%)$ for women. $^{2}$ The rates however show a range of values from $3 \%$ to $22 \%$, and prevalence is higher among vulnerable groups, such as migrants. Swedish healthcare facilities are well resourced and offer personalised support strategies for prevention and health promotion, including innovative strategies such as 'physical activity on prescription'.

SMART2D, Self-Management Approach and Reciprocal learning for Type 2 Diabetes.

Sweden. ${ }^{20}$ Acknowledgement of the gaps and strengths in each setting was used as a basis for reciprocal learning, using knowledge transfer and its application.

The main objective was to formulate and implement a contextually appropriate self-management strategy for the prevention and control of T2D in each setting and to evaluate its outcomes. Process objectives were to develop a reciprocal learning approach to increase contextual understanding and its impact on intervention development and implementation, and knowledge translation to influence national policymaking and guidelines.

In order to support knowledge translation and policy uptake, the Evidence Integration Triangle (EIT) was used to guide reciprocal learning across project phases (figure 1). The framework emphasises the interaction of evidence-based interventions, practical progress measures and participatory implementation processes, and is well suited for complex interventions with multiple components and stakeholders. ${ }^{21}$

\section{Learning cycles}

The work process in the project was structured into three phases with corresponding learning cycles, involving problem clarification and the development of a Theory of Change or ToC (formative phase); development and 


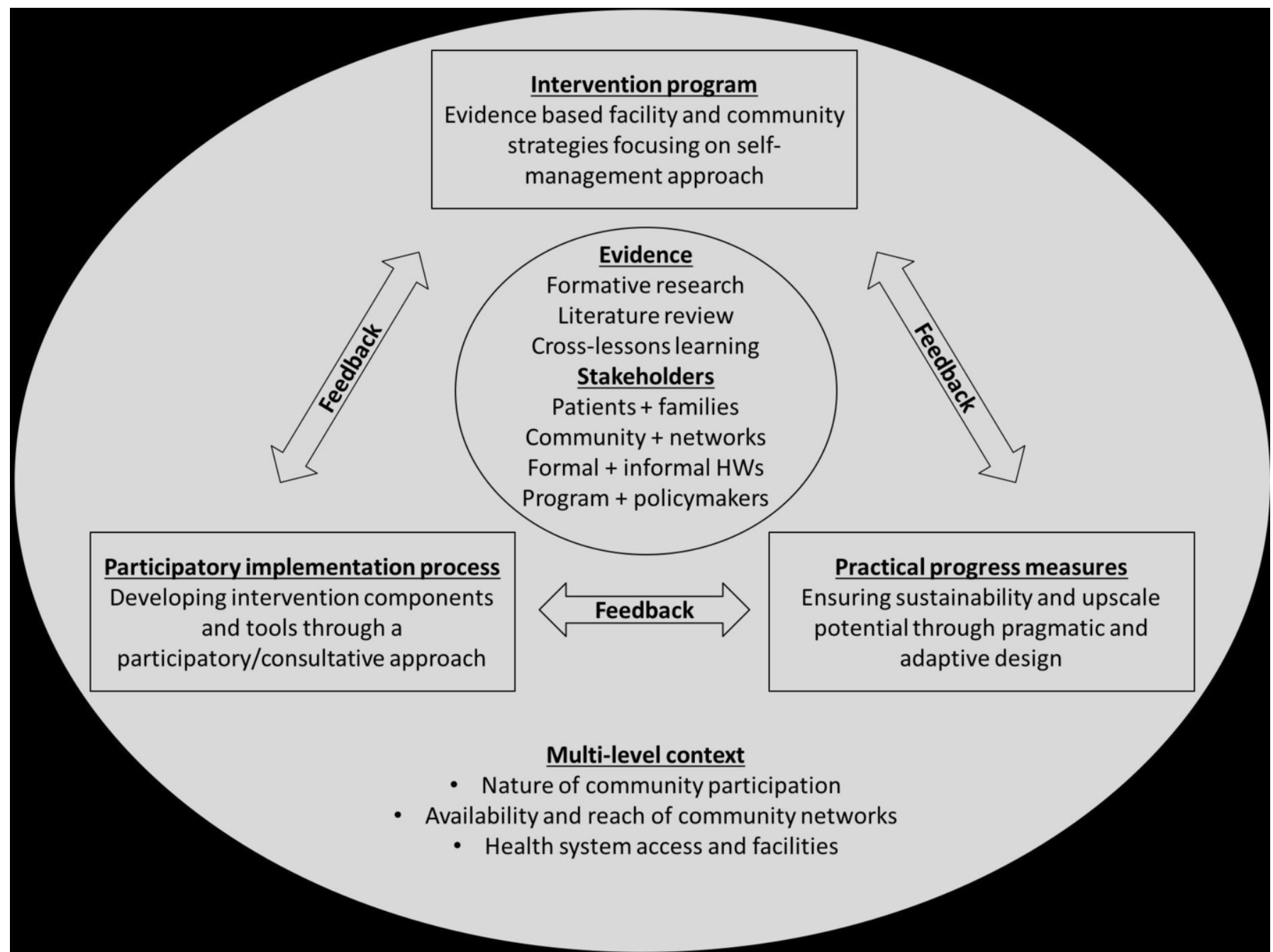

Figure 1 Evidence Integration Triangle as outlined in the SMART2D project proposal. HWs, health workers; SMART2D, SelfManagement Approach and Reciprocal learning for Type 2 Diabetes.

contextualisation of the intervention framework (intervention phase); and implementation and evaluation of the intervention (trial phase). The learning cycles partly overlapped, illustrating their interlinkages and the continuous matching of evidence and context. Each cycle was facilitated by a consortium partner and addressed elements of the EIT framework.

\section{Problem clarification and Theory of Change development}

Research has shown how a ToC can inform implementation of health systems innovations. ${ }^{22}$ In this learning cycle, problem clarification helped develop a ToC, which emphasised that diabetes self-management is influenced by the patient-provider interaction and responsiveness of health services, which in turn are influenced by the active involvement of patients and their families, professional providers, and community actors. The cycle resulted in three situational analyses that revealed opportunities and threats for potential intervention.

In the Ugandan context, the limited capacity and resource constraints of health services, coupled with insufficiently detailed care guidelines and overall lack of clarity on self-management, hindered responsive care. Opportunities for change included improving service delivery to reach minimum levels of quality care and strengthening patient involvement. In South Africa, the environment acted as a barrier to improving self-management through its impact on diet and physical activity. Opportunities for change included strengthening of community-based groups to advocate change, improving the link between community health workers (CHWs) and health facilities, and strengthening patient roles through behavioural coaching. In the Swedish site, immigrants and other disadvantaged groups experienced delays in receiving health services, limited cultural understanding and trust, and compromised patient-provider interactions in relation to self-management advice. ${ }^{23}$ Opportunities for change included establishing a link between patients and health providers, local administrations and community-based organisations to facilitate understanding, social support and trust. 
Gaps in one setting were addressed by looking at experiences from other field sites. Care models from Sweden and South Africa informed the development of algorithms, patient stratification and motivational health counselling in Uganda. In Sweden, identification of community actors and their potential role was guided by the experience of working with CHWs in South Africa and Uganda. A tool to evaluate the food environment (Environmental Profile of a Community's Health) ${ }^{24}$ was adapted in South Africa, and translated for use in Sweden and Uganda. The overall output was an improved ToC with more emphasis on the community, environment and relationships between different actors.

\section{Development and contextualisation of the intervention framework}

A generic framework was developed including common strategies for facilities (organisation of care, strengthened patient roles) and communities (community mobilisation, environmental support and community extension). Strategies were selected using situation analysis, literature review of evidence-based interventions and stakeholder workshops. The engagement with stakeholders of different levels was organised in multiple sessions, starting with an inclusive introduction workshop followed by repeated interactions with smaller groups or key individuals in the community or health system. Cross-site workshops were organised to contextualise these strategies and select optional components, which resulted in the coproduction of a common set of tools and site-specific strategies.

The Ugandan site developed guidelines for facility-based diabetes care, health education and motivational coaching, guidelines for a community-based peer support group programmes, and guidelines for care companions. The South African site produced material for peer support group activities and developed health education materials and group tools to evaluate the local food environment in collaboration with the Swedish site. The latter also developed specific tasks for the community link team and different models for community-based mobilisation and screening, based on experiences from Uganda and South Africa.

In Uganda, site-specific strategies focused on improving quality care by providing equipment and supplies, developing and implementing guidelines and task-shifting, and strengthening self-management by establishing community-based peer support groups. In South Africa, strategies for improving self-management included developing a peer group-led community walk for patients to help understand and navigate the local food environment, and involving CHWs to provide individual patient care and assist in peer group development. The Swedish site developed links between health facilities, local administration and community actors, and introduced the concept of a 'community link team' to formalise these linkages.
Implementation and evaluation of the adaptive implementation trial

This learning cycle focused on implementing and evaluating the intervention, which mainly consisted of an adaptive implementation trial. ${ }^{25}$ Dialogues with stakeholders and findings from the previous learning cycles helped refine the target population, recruitment strategies and evaluation questions for each site. A pragmatic cluster design was used, with clusters consisting of facilities and their catchment area. Inclusion criteria for study enrolment were identical across sites, but site-specific algorithms were developed to screen and recruit participants based on resource availability, current practices and national guidelines, and logistical arrangements. ${ }^{25}$ The trial is guided by a generic trial protocol, implementation guide and evaluation tools.

In Uganda, nine clusters were randomised into three study arms to compare usual with optimised facility-based care and facility-based plus community-based care. In Sweden, to facilitate trial acceptability, the target population was expanded to all adults living in selected clusters irrespective of their place of birth. Recruitment of participants revealed challenges in mobilising the target population, which shifted the focus towards evaluating implementation feasibility. In South Africa, population density and risk of study contamination resulted in selecting two clusters based on pragmatic considerations, such as implementation feasibility and avoidance of overlap with other studies.

\section{Contextualisation}

A crucial element of implementation research is the process of contextualising the intervention, ${ }^{26}$ which in the case of SMART2D took place across three different sites. The interaction with local stakeholders enabled site teams to better understand their context and consider the relevance and feasibility of interventions in their own setting. Organised stakeholder interactions in individual and group meetings also allowed to balance between efficiency in intervention development and participatory bottom-up codevelopment, which was critical to realise an implementable research agenda. Other research confirms the importance of networks and 'extra team connections' (ie, outside of site teams but within the organisation) to contribute to change, spread innovations and contribute to skills development within the network. ${ }^{27}$

The consecutive learning cycles also helped teams to understand the change process, to create a platform for knowledge exchange and shared learning, to think outside the box, and to optimise problem-solving capacity. While many multisite intervention studies emphasise intervention fidelity to allow cross-site comparison, the approach taken in this study allowed harmonisation of the intervention by function rather than content, in line with the recommendation to find balance between fidelity and local adaptation. ${ }^{28}$ The intervention strategy of 'linking the health facility with the community' was 
hence tackled differently in each setting depending on context-specific needs, for instance face-to-face meetings to receive support materials or phone calls to inform about patients.

\section{Reciprocal learning}

Reciprocal learning took place in each learning cycle through workshops, monthly teleconferences and periodic debriefings, as illustrated in table 1 and figure 2. There was a 5-day face-to-face workshop for each learning cycle, in which researchers from each country participated and which was facilitated by the person in lead of that learning cycle. The learning was facilitated by the generation and application of evidence within and across contexts, and by collaboration with local stakeholders. Outputs were described in project documents and deliverables, and the trial protocol was published as a scientific paper. ${ }^{25}$ The framework and methodologies were instrumental in maintaining the reciprocal learning focus, providing a robust yet flexible framework to structure learning in each cycle. This allowed for a balance between evidence, stakeholder needs and pragmatic contextual adaptation.

Managing the outputs of the learning process to achieve feasible strategies for implementation and evaluation, however, is complex and unpredictable, and depends to a large extent on individual commitment, relationships and team performance. Actors are crucial in shaping the collaboration, and member and team interdependence sometimes resulted in delays and friction around common goals, which required early recognition and team building to instil trust and ownership. Interpersonal processes such as communication networks, conflict resolution strategies and leadership styles influenced team readiness for collaboration and the capacity to work together effectively over extended periods of time. ${ }^{29}$ Each learning cycle lasted for 1 year and the time commitment included monthly contact moments with each country team. Content expertise on the intervention and the methods and actor autonomy also facilitated a more focused collaboration and allowed tensions to be solved more easily.

Partnerships and relationships are key to reciprocal learning, ${ }^{30}$ and in this project were found to be crucial to obtaining synergistic outcomes. Horizontal leadership and partnership strengthening using proven strategies such as joint ownership, authorship merits and celebration of achievements also contributed to creating a proactive learning environment, as reported elsewhere. ${ }^{15}{ }^{31} \mathrm{In}$ addition, partnerships can offer considerable benefits to academic institutions by way of access to resources and opportunities for individual and institutional capacity building. ${ }^{32}$ Reciprocal learning pushes these collaborations a step further, towards cocreation of knowledge and application of evidence, although more may be needed to facilitate a multidirectional flow of ideas and innovation. ${ }^{30}$
In SMART2D, the process started by identifying site-specific strengths and limitations and their potential for cross-site learning, for example the adaptation of community-linked models of healthcare from Uganda and South Africa to the Swedish site. This type of reverse innovation has been found to strengthen relationships in other cases of shared learning ${ }^{33}$ and is increasingly recognised as a potential tool for generating effective solutions to global health systems. ${ }^{34}$ The strategy has been used to help address health system challenges in HICs, including improving care for vulnerable population groups, ${ }^{34}$ a process which has been dubbed 'frugal innovation', or identifying low-cost solutions for problems affecting HICs. ${ }^{35}$

This study thus illustrates the relationship between reciprocal learning, innovation and engagement. Engagement is a condition for change and innovation can stimulate reciprocal learning. This in turn can increase engagement of researchers with the intervention; of providers with their patients; and finally of individuals (with T2D) with their illness and self-management. This virtuous cycle holds promise, enabling learning health systems to develop in each context ${ }^{36}$ and contributing to the establishment of learning networks in general. These learning networks are beneficial in promoting the development agenda, in particular to shift partnerships between LMICs and HICs from conditionality-driven, aid-dominated relationships to more integrated and collaborative efforts. ${ }^{16}$

\section{Methodological considerations}

The framework that guides the cyclical learning and its methodological components has been used previously in implementation research, both in LMICs ${ }^{37}$ and in HICs. ${ }^{38}$ As a socioecological framework, the EIT is designed for use by practitioners, policymakers and citizens to foster evidence integration through partnership learning. ${ }^{21}$ The EIT hence aligns well with patient-oriented research and quality improvement, although it may lack detailed attention to the impact of culture and society and to sustainability. However, some authors have argued that programmes are rarely successful as initially implemented and need to evolve and adapt to a changing context in order to be sustainable. ${ }^{39}$

The generalisability of study results should be considered from the view of transferability. ${ }^{40}$ The reciprocal learning approach in this study allows for tailoring an intervention and thus improving transferability to other similar cases. ${ }^{41}$ Yet the differences in outcomes between sites are the result of the interaction between intervention and context, and disentangling the attribution of both is difficult. Analysis of the adaptation in each site allows a better understanding of this entwinement and will contribute to identifying the role of context in the effectiveness of the intervention. Pragmatic trials are context-dependent, and evaluations of pragmatic studies should therefore report on the multilevel context within which such studies are conducted to allow for 
Table 1 Learning cycles and their output in terms of reciprocal learning

S no Name of output Reciprocal learning process Examples of reciprocal learning

Learning cycle 1: problem clarification and developing a ToC

1. Situational Data from formative phase.

analysis $\quad$ Online group discussions with implementation staff from each site.

- Online debriefing and synthesis.

\begin{tabular}{|c|c|c|c|}
\hline 2. & Topic guide & $\begin{array}{l}\text { Developed based on discussions related to } \\
\text { the situational analysis. } \\
\text { Scoping (grey and published) literature } \\
\text { reviews conducted in two sites and fed into } \\
\text { the topic guide. } \\
\text { Tool modified based on joint online } \\
\text { discussions with site teams led by the } \\
\text { responsible work leader. }\end{array}$ & $\begin{array}{l}\text { Common evaluation after data collection } \\
\text { led to awareness of the difficult } \\
\text { interpretation of individual behavioural } \\
\text { concepts in all contexts. }\end{array}$ \\
\hline 3. & $\begin{array}{l}\text { Theory of change } \\
\text { (ToC) }\end{array}$ & $\begin{array}{l}\text { First cross-contextual workshop. } \\
\text { Data from topic guide discussed and } \\
\text { incorporated. } \\
\text { Findings from situational analysis } \\
\text { incorporated. }\end{array}$ & $\begin{array}{l}\text { Adaptation of common ToC with more } \\
\text { emphasis on environment, linkage } \\
\text { between actors in health system and } \\
\text { community. }\end{array}$ \\
\hline
\end{tabular}

Learning cycle 2: development of the intervention framework and its contextualisation

\begin{tabular}{|c|c|c|c|}
\hline 4. & $\begin{array}{l}\text { Intervention } \\
\text { framework }\end{array}$ & $\begin{array}{l}\text { Second cross-contextual workshop. } \\
\text { Site visits by the responsible work leader. } \\
\text { Site-specific stakeholder workshops to } \\
\text { discuss implementation feasibility and } \\
\text { strategies relevant for each context. } \\
\text { Online team discussions by phone and email } \\
\text { on strategies to be included in the generic } \\
\text { model. }\end{array}$ & $\begin{array}{l}\text { Identification of core common intervention } \\
\text { strategies (organisation of care and } \\
\text { strengthening patient role in facility; } \\
\text { mobilisation, environmental support and } \\
\text { community extension in community) and } \\
\text { optional elements for each site. } \\
\text { Strategies contextualised specifically to } \\
\text { site needs and resources. }\end{array}$ \\
\hline 5. & Intervention tools & $\begin{array}{l}\text { Each country team led the development of } \\
\text { the most relevant tools, together with the } \\
\text { topic team, followed by adaptation by other } \\
\text { country teams. }\end{array}$ & $\begin{array}{l}\text { Generic draft tools and finalised site- } \\
\text { specific tools developed: patient flow } \\
\text { algorithms, peer group manual, care } \\
\text { companion guidelines, environment- } \\
\text { related interventions for peer groups. }\end{array}$ \\
\hline
\end{tabular}

Learning cycle 3: implementation and evaluation of the adaptive implementation trial

\begin{tabular}{|c|c|c|c|}
\hline 6. & $\begin{array}{l}\text { Community } \\
\text { mobilisation, } \\
\text { screening and } \\
\text { recruitment }\end{array}$ & $\begin{array}{l}\text { Third cross-contextual workshop. } \\
\text { Field testing, discussion in conference calls } \\
\text { and with trial and evaluation coordinator, } \\
\text { adaptations. }\end{array}$ & $\begin{array}{l}\text { Common development of information } \\
\text { materials. } \\
\text { Testing of different strategies, three } \\
\text { contextualised testing and recruitment } \\
\text { algorithms and strategies. }\end{array}$ \\
\hline 7. & Peer mobilisation & $\begin{array}{l}\text { Testing strategies for peer mobilisation } \\
\text { and peer leader selection and participant } \\
\text { engagement strategies, sharing in cross- } \\
\text { country conference calls. }\end{array}$ & $\begin{array}{l}\text { Peer leader training and refresher } \\
\text { strategy. } \\
\text { Contextualised models for patient } \\
\text { engagements to peer groups. }\end{array}$ \\
\hline 8. & $\begin{array}{l}\text { Data collection } \\
\text { tools }\end{array}$ & $\begin{array}{l}\text { Third cross-contextual workshop and } \\
\text { working groups. } \\
\text { Field testing, feedback and adaptation. }\end{array}$ & $\begin{array}{l}\text { Common data collection guide, with } \\
\text { context-specific addendum. }\end{array}$ \\
\hline 9. & $\begin{array}{l}\text { Process } \\
\text { evaluation tools }\end{array}$ & $\begin{array}{l}\text { Thematic cross-contextual workshop, site } \\
\text { visits. } \\
\text { First minimum set of process indicators. } \\
\text { Comprehensive protocol developed in } \\
\text { Uganda-informed other countries. }\end{array}$ & $\begin{array}{l}\text { Minimum set of process indicators } \\
\text { implemented, with country-specific } \\
\text { extensions. }\end{array}$ \\
\hline
\end{tabular}




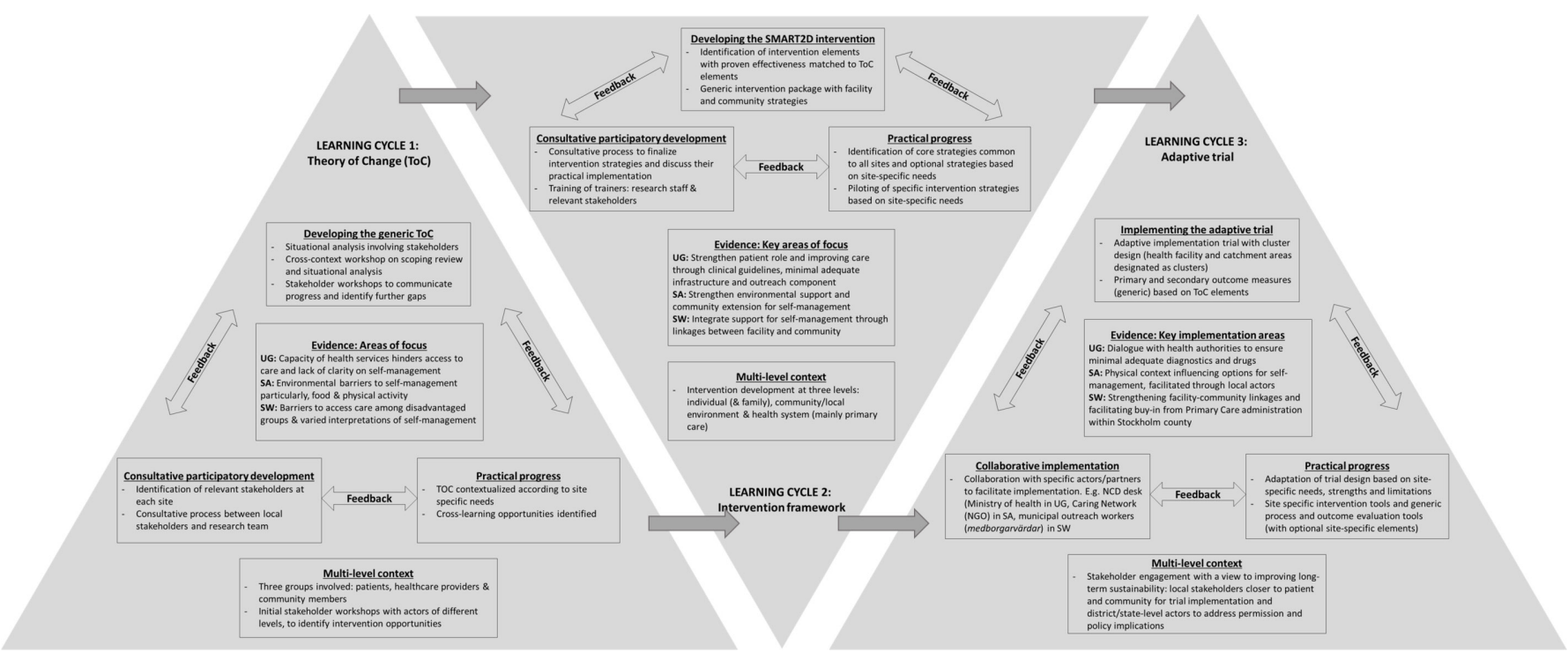

Figure 2 The three learning cycles and reciprocal learning opportunities depicted using the Evidence Integration Triangle. NCD, non-communicable disease; NGO, non-governmental organisation; SA, South Africa; SMART2D, Self-Management Approach and Reciprocal learning for Type 2 Diabetes; SW, Sweden; UG, Uganda.

potential adopters to determine the adaptability to their own setting. ${ }^{42}$

Finally, cross-cultural institutional partnerships necessitate an awareness of power dynamics. In unbalanced power contexts, the ability to exchange knowledge and resources may be compromised, leading to a partner developing a sense of distributive injustice. ${ }^{14}$ Potential conflict between partners can be counteracted by continuing to build a spirit of reciprocal commitment and trust, in turn encouraging partners to engage in democratic and participative processes of knowledge sharing. As found in this project, the relationships formed and the mutual learning that took place hence became more than 'soft' benefits. ${ }^{43}$

\section{CONCLUSION}

The reciprocal learning approach as applied in this project allowed for a structured methodology and framework for the cocreation of knowledge and advancing implementation solutions for global health problems. The approach allowed for an indepth understanding of contextual differences in improving self-management of T2D and for the adaptability of solutions. The learning culture and emphasis on cross-lessons facilitated interactions within and between partners beyond specific research activities and can be considered relevant for the wider regions (Europe and sub-Saharan Africa) represented by this collaborative partnership.

\section{PATIENT AND PUBLIC INVOLVEMENT}

Patients were involved from the first learning cycle and in all subsequent phases in the study. This was done through workshops in each site, involving collaborations with patient organisations or clubs in each country. In
Sweden, we also worked with local ethnic associations to involve people who were not (yet) identified as patients.

The discussions and testing of ideas with patients and associated groups led to the development of the intervention focused on the problems experienced by the patients. The implementation of the intervention was adapted to overcome barriers to participation. For instance, potential patients in Sweden preferred to have a 'one-stop-shop' approach to recruitment, which led to the adaptation of the procedure.

\section{Author affiliations \\ ${ }^{1}$ Department of Public Health, Institute of Tropical Medicine, Antwerp, Belgium ${ }^{2}$ Department of Primary and Interdisciplinary Care, University of Antwerp, Antwerp, Belgium \\ ${ }^{3}$ Chronic Disease Initiative for Africa, Faculty of Health Sciences - University of Cape Town, Cape Town, South Africa \\ ${ }^{4}$ School of Public Health, University of Western Cape, Bellville, South Africa ${ }^{5}$ Department of Epidemiology and Biostatistics, School of Public Health, Makerere University College of Health Sciences, Kampala, Uganda \\ ${ }^{6}$ Department of Public Health and Clinical Nutrition, University of Eastern Finland, Kuopio, Finland \\ ${ }^{7}$ Collaborative Care Systems Finland, Helsinki, Finland \\ ${ }^{8}$ School of Public Health, University of the Western Cape, Cape Town, South Africa ${ }^{9}$ Department of Public Health Sciences, Karolinska Institutet, Stockholm, Sweden ${ }^{10}$ Department of Molecular Medicine and Surgery, Diabetes and Endocrine Unit, Karolinska Institutet, Stockholm, Sweden \\ ${ }^{11}$ Department of Public Health Sciences, Karolinska Institutet and Swedish Institute for Global Health Transformation (SIGHT), Stockholm, Sweden \\ ${ }^{12}$ Department of Learning, Informatics, Management and Ethics, Karolinska Institutet, Stockholm, Sweden \\ ${ }^{13}$ Department of Physiology \& Pharmacology, Karolinska Institutet, Stockholm, Sweden \\ ${ }^{14}$ Uppsala University, Uppsala, Sweden \\ ${ }^{15}$ Dept of Food Studies, Nutrition and Dietetics, Uppsala University, Uppsala, Sweden}

Acknowledgements The SMART2D consortium includes the authors of this paper. We also acknowledge the contributions of other researchers of the SMART2D 
consortium, including Elizabeth Ekirapa Kiracho, Juliet Aweko, Juliet Kiguli and Linda Timm.

Contributors Jv0, PD and MD conceptualised the format of this paper and developed the several drafts. SP gave conceptual input to the development of the study and of the paper. DG, GT and PA provided textual and structural comments to the different versions of the manuscript. CG-0, DS, CJS and TP participated in the development of the approach and gave comments on the manuscript. All authors read and approved the final version of this manuscript.

Funding This work was supported by the European Commission's Horizon 2020 Health Coordination Activities (Grant Agreement No 643692) under call 'HCO-052014: Global Alliance for Chronic Diseases: prevention and treatment of type 2 diabetes'. The content of this article is solely the responsibility of the authors and does not reflect the views of the EU.

Competing interests None declared.

Patient consent Not required.

Ethics approval The study was approved by the ethics committee in each of the respective countries, including the Higher Degrees, Research and Ethics Committee of Makerere University School of Public Health and the Uganda National Council for Science and Technology (Ref HDREC-309 and HS 1894, respectively) in Uganda; the Office of the Dean, Department of Research Development (Ref 15/3/17) of the University of Western Cape, South Africa; the Regional Ethics Review Board in Stockholm (Ref 2015/712-31/1), Sweden; and the Institutional Review Board (Ref 993/14), Institute of Tropical Medicine, Belgium. Patients participating in the trial have signed informed consent to participate in the trial.

Provenance and peer review Not commissioned; externally peer reviewed.

Data sharing statement The data sets used and/or analysed during the current study are available from the corresponding author on reasonable request.

Open access This is an open access article distributed in accordance with the Creative Commons Attribution Non Commercial (CC BY-NC 4.0) license, which permits others to distribute, remix, adapt, build upon this work non-commercially, and license their derivative works on different terms, provided the original work is properly cited, appropriate credit is given, any changes made indicated, and the use is non-commercial. See: http://creativecommons.org/licenses/by-nc/4.0

\section{REFERENCES}

1. United NationsGeneral Assembly Resolution. A/RES/70/1. Transforming Our World: the 2030 Agenda for Sustainable Development, 2015

2. NCD Risk Factor Collaboration (NCD-RisC). Worldwide trends in diabetes since 1980: a pooled analysis of 751 population-based studies with 4.4 million participants. Lancet 2016;387:1513-30.

3. Dagenais GR, Gerstein HC, Zhang X, et al. variations in diabetes prevalence in low-, middle-, and high-income countries: results from the prospective urban and rural epidemiological study. Diabetes Care 2016;39:780-7.

4. Walker RJ, Strom Williams J, Egede LE. influence of race, ethnicity and social determinants of health on diabetes outcomes. Am J Med Sci 2016;351:366-73.

5. Gilson L, Doherty J, Loewenson R. Final report of the Knowledge Network on health systems. In: Challenging inequity through health systems, 2007

6. American Diabetes Association (ADA). Standards of medical care in diabetes - 2017. Diabetes Care 2017;40(S1):s4-s128.

7. Grover A, Joshi A. An overview of chronic disease models: a systematic literature review. Glob J Health Sci 2014;7:210-27.

8. Funnell MM, Brown TL, Childs BP, et al. National standards for diabetes self-management education. Diabetes Care 2012;35(Suppl 1):S101-S108.

9. Bukhman G, Kidder A. The PIH Guide to Chronic Care Integration for Endemic Non-Communicable Diseases. Boston, MA: Partners in Health, 2011: 153-85.

10. Fisher EB, Earp JA, Maman S, et al. Cross-cultural and international adaptation of peer support for diabetes management. Fam Pract 2010;27(Suppl 1):i6-i16.

11. Binagwaho A, Nutt CT, Mutabazi V, et al. Shared learning in an interconnected world: innovations to advance global health equity. Global Health 2013;9:37.

12. Cars $\mathrm{O}, \mathrm{Xiao} Y$, Stålsby Lundborg $\mathrm{C}$, et al. Building bridges to operationalise one health - A Sino-Swedish collaboration to tackle antibiotic resistance. One Health 2016;2:139-43.
13. Silver H, Strong R, Perini M. The strategic teacher: Selecting the right research-based strategy for every lesson. Alexandria, Virginia: Association for Supervision and Curriculum Development, 2007.

14. Muthusamy SK, White MA. Learning and knowledge transfer in strategic alliances: A social exchange view. Organization Studies 2005;26:415-41.

15. Hagen NA, Stiles CR, Biondo PD, et al. Establishing a multicentre clinical research network: lessons learned. Curr Oncol 2011;18:e243-9.

16. Kulasabanathan $\mathrm{K}$, Issa $\mathrm{H}$, Bhatti $\mathrm{Y}$, et al. Do International Health Partnerships contribute to reverse innovation? a mixed methods study of THET-supported partnerships in the UK. Global Health 2017;13:25.

17. Asiimwe S, Ross JM, Arinaitwe A, et al. Expanding HIV testing and linkage to care in southwestern Uganda with community health extension workers. J Int AIDS Soc 2017;20(Suppl 4):21633.

18. Grimsrud A, Sharp J, Kalombo C, et al. Implementation of community-based adherence clubs for stable antiretroviral therapy patients in Cape Town, South Africa. J Int AIDS Soc 2015;18:19984.

19. Mahomed $\mathrm{OH}$, Asmall S, Freeman M. An integrated chronic disease management model: a diagonal approach to health system strengthening in South Africa. J Health Care Poor Underserved 2014;25:1723-9.

20. National Board of Health and Welfare, 2014. Quality and efficiency of diabetes care in Sweden: National Performance Assessment 2011.Available from:https://www.socialstyrelsen.se/Lists/ Artikelkatalog/Attachments/19393/2014-3-18.pdf [Accessed1 Jul 2018].

21. Glasgow RE, Green LW, Taylor MV, et al. An evidence integration triangle for aligning science with policy and practice. Am J Prev Med 2012;42:646-54.

22. Paina L, Wilkinson A, Tetui M, et al. Using Theories of Change to inform implementation of health systems research and innovation: experiences of Future Health Systems consortium partners in Bangladesh, India and Uganda. Health Res Policy Syst 2017;15(Suppl 2):109.

23. Daivadanam M, Östenson C-G, Alvesson HM, et al. Selfmanagement perceptions among providers and non-European type 2 diabetes patients in Stockholm. Eur J Public Health 2017;27(suppl_3):265-6.

24. Chow CK, Lock K, Madhavan M et al. Environmental Profile of a Community's Health (EPOCH): an instrument to measure environmental determinants of cardiovascular health in five countries. PLoS One 2010;5:e14294.

25. Guwatudde D, Absetz P, Delobelle P, et al. Study protocol for the SMART2D adaptive implementation trial: a cluster randomised trial comparing facility-only care with integrated facility and community care to improve type 2 diabetes outcomes in Uganda, South Africa and Sweden. BMJ Open 2018;8:e019981.

26. Edwards N, Barker PM. The importance of context in implementation research. J Acquir Immune Defic Syndr 2014;67 Suppl 2(Suppl 2):S157-S162.

27. Ramanadhan S, Wiecha JL, Emmons KM, et al. Extra-team connections for knowledge transfer between staff teams. Health Educ Res 2009;24:967-76.

28. Brown $\mathrm{CH}$, Curran G, Palinkas LA, et al. An overview of research and evaluation designs for dissemination and implementation. Annu Rev Public Health 2017;38:1-22.

29. Gray B. Enhancing transdisciplinary research through collaborative leadership. Am J Prev Med 2008;35(2 Suppl):S124-S132.

30. Issa $H$, Kulasabanathan K, Darzi A, et al. Shared learning in an interconnected world: the role of international health partnerships. $J$ $R$ Soc Med 2017;110:316-9.

31. Sewankambo N, Tumwine JK, Tomson G, et al. Enabling dynamic partnerships through joint degrees between low- and high-income countries for capacity development in global health research: experience from the Karolinska Institutet/Makerere University partnership. PLoS Med 2015;12:e1001784.

32. Muir J, Farley J, Osterman A. Global health programs and partnerships: Evidence of mutual benefit and equity. Washington, DC: CSIS Global Health Policy Center, 2016.

33. Johnson CD, Noyes J, Haines A, et al. Learning from the Brazilian community health worker model in North Wales. Global Health 2013;9:25.

34. Syed SB, Dadwal V, Rutter P, et al. Developed-developing country partnerships: benefits to developed countries? Global Health 2012;8:17.

35. Bhatti YA, Prime M, Harris $M$, et al. The search for the holy grail: frugal innovation in healthcare from low-income or middle-income countries for reverse innovation to developed countries. BMJ Innov 2017:3:212-20. 
36. Greene SM, Reid RJ, Larson EB. Implementing the learning health system: from concept to action. Ann Intern Med 2012;157:207-10.

37. Sivaram S, Sanchez MA, Rimer BK, et al. Implementation science in cancer prevention and control: a framework for research and programs in low- and middle-income countries. Cancer Epidemiol Biomarkers Prev 2014;23:2273-84.

38. Billings $\mathrm{J}$. What works for whom, how, in what context and with what outcomes? Integrated care evaluation using the Evidence Integration Triangle: the case of SUSTAIN. Int J Integr Care 2016;16:A125

39. Glasgow RE, Chambers D. Developing robust, sustainable, implementation systems using rigorous, rapid and relevant science. Clin Trans/ Sci 2012;5:48-55.

40. Polit DF, Beck CT. Generalization in quantitative and qualitative research: myths and strategies. Int J Nurs Stud 2010;47:1451-8.

41. Lewis CC, Klasnja P, Powell BJ, et al. From classification to causality: advancing understanding of mechanisms of change in implementation science. Front Public Health 2018;6:136.

42. Glasgow RE. What does it mean to be pragmatic? Pragmatic methods, measures, and models to facilitate research translation. Health Educ Behav 2013;40:257-65.

43. Boydell LR, Rugkåsa J. Benefits of working in partnership: a model. Crit Public Health 2007:17:217-28.
44. Bahendeka S, Wesonga R, Mutungi G, et al. Prevalence and correlates of diabetes mellitus in Uganda: a population-based national survey. Trop Med Int Health 2016;21:405-16.

45. NCD Risk Factor Collaboration (NCD-RisC) - Africa Working Group. Trends in obesity and diabetes across Africa from 1980 to 2014: an analysis of pooled population-based studies. Int J Epidemiol 2017;46:1421-32.

46. Fisher EB, Boothroyd RI, Coufal MM, et al. Peer support for self-management of diabetes improved outcomes in international settings. Health Aff 2012;31:130-9.

47. Peer N, Steyn K, Lombard C, et al. Rising diabetes prevalence among urban-dwelling black South Africans. PLoS One 2012; 7:e43336.

48. Western Cape Government Health. Primary health care: integrated audit report for non-communicable chronic diseases, Cape Town district 2016/17. Cape Town: Western Cape Department of Health, 2017.

49. . The 2012 SEMDSA Guideline for the Management of Type 2 Diabetes (Revised). J Endocrinol Metabol Diabetes S Af 2012;17:S1-S95

50. Igbojiaku OJ, Harbor OC, Ross A. Compliance with diabetes guidelines at a regional hospital in KwaZulu-Natal, South Africa. Afr J Prim Health Care Fam Med 2013;5. 